\title{
Group index dispersion of holey fibres measured by a white-light spectral interferometric technique
}

\author{
P. Hlubina* , D. Ciprian, R. Chlebus \\ Department of Physics, Technical University Ostrava, 17. listopadu 15, \\ 70833 Ostrava-Poruba, Czech Republic
}

\begin{abstract}
We present a new white-light interferometric technique to measure the group index of holey fibres over a wide wavelength range. The technique utilizes an unbalanced Mach-Zehnder interferometer with a fibre under test of known length placed in one of the interferometer arms and the other arm with adjustable path length. In a first step, the differential group index of the fibre is measured over a wide wavelength range. In a second step, the fibre is replaced by the reference sample of known thickness and group dispersion to determine precisely the group index of the fibre at one specific wavelength. The group index as a function of wavelength is measured for two different holey fibres, one made of pure silica glass and the other made of SK222 glass. For both fibres, the wavelength dependence of the group index of the outer cladding and modes supported by the fibre is measured.
\end{abstract}

Key words: spectral interferometry, white-light source, Mach-Zehnder interferometer, holey fibre, group index, dispersion, pure silica glass, SK222 glass PACS: 07.60, 42.25.Hz, 42.70.Ce, 42.81.Cn, 78.20.Ci

\section{Introduction}

A precise measurement of the wavelength dependence of the group refractive index (group index dispersion) of optical fibres over a broad spectral range is important in various research areas including high-speed optical transmission systems and broadband optical communications. The chromatic dispersion, which can be obtained by simply differentiating the differential group index in

* Tel.: +420-597-323-134; fax: +420-597-323-139.
Email address: petr.hlubina@vsb.cz (P. Hlubina). 
the the spectral domain, is a significant characteristic that affects the bandwidth of a high-speed optical transmission system through pulse broadening and nonlinear optical distortion. Chromatic dispersion of long length optical fibres is determined by two widely used methods [1]: the time-of-flight method, which measures relative temporal delays for pulses at different wavelengths, and the modulation phase shift technique, which measures the phase delay of a modulated signal as a function of wavelength.

White-light interferometry based on the use of a broadband source in combination with a standard Michelson or Mach-Zehnder interferometer [2] is considered as one of the best tools for dispersion characterization of short length optical fibres. White-light interferometry usually utilizes a temporal method or a spectral method. The temporal method involves measurement of the group delay introduced by an optical fibre which is placed in one of the interferometer arms and evaluating the temporal shift of the peak of the cross-correlation interferogram. As the central wavelength is varied, the relative group delay of different frequency components is observed directly [3]. Alternatively, the spectral distribution of the phase delay over the full bandwidth of the whitelight source is obtained in a single measurement by a Fourier transform of the cross-correlation interferogram [4]. The dispersion characteristics of the fiber sample under study can be obtained by simply differentiating the measured phase delay.

The spectral method is based on the observation of spectrally resolved interference fringes (channelled spectrum) in the vicinity [5-7] of a stationary phase point or far from it [8] and involves measurement of the phase or period of the spectral fringes. The group dispersion of a fibre sample can be obtained by simply differentiating the spectral phase retrieved from a single interferogram. The stationary phase point appears in the recorded spectral interferogram when the overall group optical path difference (OPD) between two beams in the interferometer is close to zero. The main limitation of this method is related to the fact that the spectral interference fringes far from the stationary phase point [8] are difficult to resolve. Using a low-resolution spectrometer [9], the measurement of the group refractive index dispersion of a given fibre is still possible in the vicinity of the stationary phase point if one moves it in successive steps to different wavelengths and repeats the measurement. The modification of the technique with a tandem configuration of a Michelson interferometer and an optical fibre has been used in measurement of the group dispersion in birefringent fibres [10-12] of known lengths. Recently, the use of the method with a Mach-Zehnder interferometer was extended for dispersion characterization of tapered fibres [7] or glasses of optical fibres [13].

The feasibility of the interferometric techniques has been demonstrated in measuring the dispersion in microstructured and holey fibres [14-17]. Both fibre-optic [15] and bulk-optic [16,17] implementations of a Michelson $[15,16]$ 
or Mach-Zehnder [17] interferometer were utilized. The dispersion parameters were obtained either by fitting the measured channelled spectrum to wavelength when the location of the stationary phase point was adjusted by the path length in the air [15] or by measuring the location of the stationary phase point (the equalization wavelength [17]) as a function of the path length difference $[16,17]$. The most recent paper [17] describes a new method of measuring the group dispersion of a holey fibre based on an apparent path length discrimination between the spectral interference fringes associated with the fundamental mode supported by the fibre and light guided by the fibre cladding the group dispersion of which is known.

The aim of this paper is to present a new white-light interferometric technique for measurement of the group index of holey fibres over a wide wavelength range. The technique utilizes an unbalanced Mach-Zehnder interferometer with a fibre under test of known length placed in one of the interferometer arms and the other arm with adjustable path length. A series of spectral interferograms is recorded to measure the equalization wavelength as a function of the path length difference. First, the differential group index of the fibre is measured over a wide wavelength range. Second, the fibre is replaced by the reference sample of known thickness and group dispersion to determine precisely the group index of the fibre under test at one specific wavelength. The group index as a function of the wavelength is measured for two different holey fibres, one made of pure silica glass and the other made of SK222 glass. For both cases, the wavelength dependence of the group index of the outer cladding and modes supported by the fibre is measured with a precision of $13 \times 10^{-5}$.

\section{Experimental method}

First, let us consider a technique presented in a previous paper [13] that has been used for measuring the differential group index $\Delta N(\lambda)$ of a fibre under test of length $z$ and refractive index $n(\lambda)$. The technique utilizes an unbalanced Mach-Zehnder interferometer (see Fig. 1) with optical components (lens 1 and lens 2) to which the effective thickness $d$ and refractive index $n_{c}(\lambda)$ correspond. The fibre under test is placed in the first (test) arm of the interferometer and the other (reference) arm is with the adjustable path length $L$ in the air so that the group OPD $\Delta_{\mathrm{MZ}}^{\mathrm{g}}(\lambda)$ between the beams in the interferometer is given by

$$
\Delta_{\mathrm{MZ}}^{\mathrm{g}}(\lambda)=L-l-N(\lambda) z-N_{c}(\lambda) d,
$$


where $l$ is the path length in the air in the test arm, and $N(\lambda)$ and $N_{c}(\lambda)$ are the group refractive indices satisfying the relation

$$
N(\lambda)=n(\lambda)-\lambda \frac{\mathrm{d} n(\lambda)}{\mathrm{d} \lambda}
$$

The spectral interference fringes recorded in the set-up have the largest period in the vicinity of a stationary phase point for which the group OPD is zero at one specific wavelength, the equalization wavelength $\lambda_{0}$. To measure the differential group index $\Delta N\left(\lambda_{0}\right)=N\left(\lambda_{0}\right)-N\left(\lambda_{0 r}\right)$ of the fiber [13], the overall path length $L=L_{o}=L_{o}\left(\lambda_{0}\right)$ has to be adjusted for which the equalization wavelength $\lambda_{0}$ is resolved in the recorded spectrum. If we choose one of the equalization wavelengths, $\lambda_{0 r}$, as the reference one, the overall path length $L_{o}\left(\lambda_{0 r}\right)$ that results from the condition $\Delta_{\mathrm{MZ}}^{\mathrm{g}}\left(\lambda_{0 r}\right)=0$ is given by

$$
L_{o}\left(\lambda_{0 r}\right)=N\left(\lambda_{0 r}\right) z+N_{c}\left(\lambda_{0 r}\right) d+l \text {. }
$$

The path length is the basis of the measurement of the fibre group dispersion $N=N\left(\lambda_{0}\right)$ from the measured differential group index dispersion $\Delta N\left(\lambda_{0}\right)=$ $N\left(\lambda_{0}\right)-N\left(\lambda_{0 r}\right)$ [13] and the group index $N\left(\lambda_{0 r}\right)$ determined precisely by another technique.

Let us consider three different techniques with three different reference elements that can be used for measuring the fibre group index $N\left(\lambda_{0 r}\right)$.

\subsection{First reference element: glass sample}

In this technique we consider a set-up of the unbalanced Mach-Zehnder interferometer in which the fibre under test is replaced by a glass sample of known thickness $t$ and group refractive index $N_{r}\left(\lambda_{0 r}\right)$ (see Fig. 1). To resolve the reference equalization wavelength $\lambda_{0 r}$ in the recorded spectrum, the reference path length $L=L_{r}=L_{r}\left(\lambda_{0 r}\right)$ have to be adjusted which satisfies the relation

$$
L_{r}\left(\lambda_{0 r}\right)=N_{r}\left(\lambda_{0 r}\right) t+N_{c}\left(\lambda_{0 r}\right) d+l-t+z .
$$

By combining Eq. (3) with Eq. (4), we obtain for the group refractive index $N\left(\lambda_{0 r}\right)$ of the fiber the relation

$$
N\left(\lambda_{0 r}\right)=1+\left[N_{r}\left(\lambda_{0 r}\right)-1\right] t / z+\left[L_{o}\left(\lambda_{0 r}\right)-L_{r}\left(\lambda_{0 r}\right)\right] / z .
$$




\subsection{Second reference element: fibre}

In this technique we consider a set-up of the unbalanced Mach-Zehnder interferometer (see Fig. 1) in which the fibre under test is replaced by the reference fibre of length $t$ and known group refractive index $N_{r}\left(\lambda_{0 r}\right)$. If the reference equalization wavelength $\lambda_{0 r}$ is resolved in the recorded spectrum for the reference path length $L_{r}\left(\lambda_{0 r}\right)$, the group refractive index $N\left(\lambda_{0 r}\right)$ of the fiber under test is given by Eq. (5).

\subsection{Third reference element: outer fibre cladding}

In this technique we consider a set-up of the unbalanced Mach-Zehnder interferometer (see Fig. 1) with the fibre under test of length $z$. If the fibre is excited in such a way that light is guided by the outer fibre cladding of known group index $N_{r}\left(\lambda_{0 r}\right)$ and if the reference equalization wavelength $\lambda_{0 r}$ is resolved in the recorded spectrum for the reference path length $L_{r}\left(\lambda_{0 r}\right)$, the group index $N\left(\lambda_{0 r}\right)$ of the fiber under test is given by

$$
N\left(\lambda_{0 r}\right)=N_{r}\left(\lambda_{0 r}\right)+\left[L_{o}\left(\lambda_{0 r}\right)-L_{r}\left(\lambda_{0 r}\right)\right] / z .
$$

To summarize the implementation merits of the above methods, the simplest is the third one. However in this case we need to know the group dispersion of the outer fibre cladding. In the opposite case, the second method is simpler than the first one if the fibre of known dispersion is available.

\section{$3 \quad$ Experimental set-ups}

The experimental set-up used in the application of spectral-domain white-light interferometry for measuring the differential group index dispersion $\Delta N=$ $\Delta N\left(\lambda_{0}\right)$ of optical fibres is shown in Fig. 1. It consists of a white-light source: a quartz-tungsten-halogen lamp (HL-2000HP, Ocean Optics, Inc.) with launching optics, a single-mode optical fibre (FS-SN-3224, 3M), a collimating lens, a bulk-optic Mach-Zehnder interferometer with plate beam splitters (BSW07, Thorlabs), a micropositioner connected to mirrors 3 and 4 of the interferometer, an aperture, a Glan-Taylor polarizer (Thorlabs), a microscope objective, micropositioners, a fibre-optic spectrometer (S2000, Ocean Optics, Inc.), an $\mathrm{A} / \mathrm{D}$ converter and a personal computer. The spectrometer resolution is given by a $50 \mu \mathrm{m}$ core diameter of the read optical fibre to which a Gaussian response function with the width of about $3 \mathrm{~nm}$ corresponds [9]. In the test 
arm of the interferometer is placed a combination of a fiber under test (fibre sample) and optical components (shown schematically in Fig. 1 as lens 1 and lens 2) represented by a microscope objective $(10 \times / 0.30$, Meopta $)$ and an achromatic lens (74-ACR, Ocean Optics, Inc.). We measured two different fibre samples. The first sample is pure silica holey fibre of length $z=50650 \mu \mathrm{m}$ (PM-1550-01, Thorlabs, see its SEM photograph in [12]). The second sample is a rectangular-shape highly birefringent holey fibre of length $z=54200 \mu \mathrm{m}$ made of SK222 optical glass with rectangular lattice and circular holes (see a similar fibre of IEMT with elliptical holes in [18]). The fibre lengths were measured by a micrometer with an accuracy of $\pm 10 \mu \mathrm{m}$.

The experimental set-up used in the application of spectral-domain whitelight interferometry for measuring the group index $N\left(\lambda_{0 r}\right)$ with the reference element is also shown in Fig. 1. The reference element is represented by a birefringent quartz plate of thickness $t=(25750 \pm 10) \mu \mathrm{m}$. The quartz plate consists of two polished surfaces, parallel to the optical axis of the crystal with a precision of 15 arcmin. The plate is inserted into the test arm of the interferometer in such a way that the collimated beam is incident on the surfaces perpendicularly. The reference group index $N_{r}\left(\lambda_{0 r}\right)$ is either $N_{o}\left(\lambda_{0 r}\right)$ or $N_{e}\left(\lambda_{0 r}\right)$ depending on the orientation of the polarizer in the set-up and subscripts $o$ and $e$ stand for the ordinary and extraordinary waves propagating in the crystal.

\section{Experimental results and discussion}

Prior to the group dispersion measurements we utilized the main advantage of the set-ups, which is in fibre connection of a light source (that can be varied) with the interferometer. We used a laser diode instead of the halogen lamp to check the precise placement and alignment of the optical components in the test arm by observing the interference fringes. Moreover, the excitation of the outer fibre cladding or core was easily inspected at the output of the test arm. In the case of the first fibre sample, the ring-shape optical field indicated that the light was guided by the outer fibre cladding [13]. Similarly, the ellipticalshape optical field indicated that the light was guided by the fiber core and the fundamental mode was supported. The orientation of the polarizer was along the longer or shorter axis of the elliptical core so that the group dispersion in the $X$ or $Y$ polarization [12] was measured.

In the dispersion measurement of the outer cladding and the fibre mode, such a path length in the reference arm of the interferometer was adjusted to resolve spectral interference fringes. Fig. 2 shows an example of the recorded normalized spectral signal [13] corresponding to the excitation of the outer fibre cladding (denoted as F+OCs). It clearly shows the effect of the limiting 
resolving power of the spectrometer on the visibility of the spectral interference fringes identified only in the vicinity of the equalization wavelength $\lambda_{0}=748.23 \mathrm{~nm}$. Fig. 2 also shows an example of the recorded spectral signal corresponding to the excitation of the fundamental mode (denoted as $\mathrm{M}+\mathrm{OCs}$ ) in the $X$ polarization with the spectral interference fringes identified only in the vicinity of the equalization wavelength $\lambda_{0}=664.54 \mathrm{~nm}$.

We measured the dependence of the adjusted path length difference on the equalization wavelength for both cases. We displaced the stage with mirrors 3 and 4 manually by using the micropositioner with a constant step of $10 \mu \mathrm{m}$ and performed recording of the corresponding spectral signals. The spectral signals recorded for the outer fibre cladding revealed that the equalization wavelength $\lambda_{0}$ can be resolved in the spectral range from 508 to $910 \mathrm{~nm}$ [13]. Similarly, the spectral signals recorded for the fundamental mode revealed that the equalization wavelength $\lambda_{0}$ can be resolved in the spectral range from 515 to $807 \mathrm{~nm}$. Knowledge of the measured dependences and the fibre length $z$ enables us to evaluate directly the differential group index $\Delta N\left(\lambda_{0}\right)$ as a function of the equalization wavelength $\lambda_{0}$ [13]. Fig. 3 shows the function for the outer cladding by the crosses (denoted as pure silica) together with the theoretical function resulting from the Sellmeier formula for pure silica [19]. This figure confirms very good agreement between theory and experiment. Similarly, Fig. 3 shows the function for the fundamental mode by the crosses (denoted as mode) and demonstrates different dispersion slope for the mode in the comparison with that for the pure silica. It should be noted here that no apparent discrimination between the spectral signals for the orthogonal polarizations of the mode was revealed in the measured spectral range. This is owing to short length of the optical fibre used in the experiment.

To measure the fibre group index $N\left(\lambda_{0 r}\right)$ at the reference equalization wavelength $\lambda_{0 r}$, first, the procedure presented in section 2.1. was used. It is important to insert the quartz crystal into the test arm of the interferometer (see Fig. 1) in order not to shift the interference pattern with respect to the centre of the aperture. Similar procedure needs to be applied in the alignment of the optical components after removing the fibre from the test arm of the interferometer. Then, such a path length $L_{r}\left(\lambda_{0 r}\right)$ in the reference arm of the interferometer was adjusted to resolve spectral interference fringes at the reference equalization wavelength $\lambda_{0 r}$. In our case, when $\lambda_{0 r}=693.62 \mathrm{~nm}$ was chosen, we adjusted $\Delta L\left(\lambda_{0 r}\right)=L_{o}\left(\lambda_{0 r}\right)-L_{r}\left(\lambda_{0 r}\right)=9348 \mu \mathrm{m}$. Using $N_{r}\left(\lambda_{0 r}\right)=N_{e}\left(\lambda_{0 r}\right)=1.56904$ resulting from dispersion relation for the birefringent quartz [20] and Eq. (5), we obtain $N_{x}\left(\lambda_{0 r}\right)=1.47386$. From the value and the measured values of the differential group index $\Delta N_{x}\left(\lambda_{0}\right)$, the group index $N_{x}\left(\lambda_{0}\right)$ of the fundamental mode as a function of the equalization wavelength $\lambda_{0}$ was determined. The function is represented in Fig. 4 by the crosses and is shown together with the function for the pure silica (crosses with the solid line). This figure shows that the group index of the fundamental mode 
is substantially higher than that of the pure silica.

We can estimate a precision of the group index measurement. If the reference equalization wavelength $\lambda_{0 r}$ is determined with an error of $\delta\left(\lambda_{0 r}\right)$, the path length difference $\Delta L$ is adjusted with a precision of $\delta(\Delta L)$ and the lengths $z$ and $t$ of the fibre and crystal are known with precisions of $\delta(z)$ and $\delta(t)$, respectively, the group index $N$ is obtained with a precision given by the following formula

$$
\begin{aligned}
\delta^{2}(N) & =\left[\frac{\mathrm{d} N_{r}\left(\lambda_{0 r}\right)}{\mathrm{d} \lambda_{0 r}} \frac{t}{z} \delta\left(\lambda_{0 r}\right)\right]^{2}+\left[\left(N_{r}-1\right) \frac{\delta(t)}{z}\right]^{2}+\left[\left(N_{r}-1\right) t \frac{\delta(z)}{z^{2}}\right]^{2} \\
& +\left[\frac{\delta(\Delta L)}{z}\right]^{2}+\left[\Delta L \frac{\delta(z)}{z^{2}}\right]^{2} .
\end{aligned}
$$

In our case, the error $\delta\left(\lambda_{0 r}\right)$ is given by the wavelength difference corresponding to adjacent pixels of the spectrometer linear CCD-array detector and is $0.32 \mathrm{~nm}$, the precision $\delta(\Delta L)$ is $1 \mu \mathrm{m}$ and the precisions $\delta(z)$ and $\delta(t)$ are $10 \mu \mathrm{m}$ so that the precision $\delta(N)$ in determining the group index is $13 \times 10^{-5}$. Higher measurement precision can be achieved using more precisely determined lengths and/or a longer fibre sample. However, there exists maximum length of the fibre given by the limited resolving power of the spectrometer.

The group index $N\left(\lambda_{0 r}\right)$ of the fundamental mode at the reference equalization wavelength $\lambda_{0 r}$ was also measured by the procedure presented in section 2.3. In this case, the excitation of the fundamental mode was changed to that of the outer cladding, the group index $N_{r}\left(\lambda_{0 r}\right)$ of which is known. Simultaneously, path length $L_{o}\left(\lambda_{0 r}\right)$ in the reference arm of the interferometer was changed to $L_{r}\left(\lambda_{0 r}\right)$ and spectral interference fringes were resolved at the same reference equalization wavelength $\lambda_{0 r}$. In our case, when $\lambda_{0 r}=693.62 \mathrm{~nm}$ was chosen, $\Delta L\left(\lambda_{0 r}\right)=118 \mu \mathrm{m}$ was adjusted. Using $N_{r}\left(\lambda_{0 r}\right)=1.47158$ resulting from dispersion relation for the pure silica [19] and Eq. (6), we obtain $N_{x}\left(\lambda_{0 r}\right)=$ 1.47391. This value is in very good agreement with that obtained by a previous procedure. Also in this case we can estimate a precision of the group index measurement, which is given by the following formula

$$
\delta^{2}(N)=\left[\frac{\mathrm{d} N_{r}\left(\lambda_{0 r}\right)}{\mathrm{d} \lambda_{0 r}} \delta\left(\lambda_{0 r}\right)\right]^{2}+\left[\frac{\delta(\Delta L)}{z}\right]^{2}+\left[\Delta L \frac{\delta(z)}{z^{2}}\right]^{2}
$$

The precision $\delta(N)$ in determining the group index by the procedure is $3 \times 10^{-5}$ and is higher than that of the previous procedure.

Finally we measured the group dispersion of the second fibre sample. The excitation of the outer fibre cladding or core was once again easily inspected at the output of the test arm. The rectangular-shape optical field indicated 
that the light was guided by the outer fibre cladding. Similarly, the ellipticalshape optical field indicated that the light was guided by the fiber core and the fibre mode was supported. The orientation of the polarizer was along the longer or shorter axis of the elliptical core so that the group dispersion in the $X$ or $Y$ polarization was measured. Fig. 5 shows an example of the recorded spectral signal (denoted as $\mathrm{F}+\mathrm{OCs}$ ), which corresponds to the excitation of the outer fibre cladding with the spectral interference fringes identified only in the vicinity of the equalization wavelength $\lambda_{0}=747.91 \mathrm{~nm}$ [13]. Fig. 5 also shows an example of the spectral signal (denoted as $X \mathrm{M}+\mathrm{OCs}$ ), which corresponds to the excitation of the fibre mode in the $X$ polarization with the spectral interference fringes identified only in the vicinity of the equalization wavelength $\lambda_{0}=607.34 \mathrm{~nm}$. Moreover, Fig. 5 shows an example of the spectral signal (denoted as $Y \mathrm{M}+\mathrm{OCs}$ ) corresponding to the excitation of the fibre mode in the $Y$ polarization with the spectral interference fringes identified only in the vicinity of the equalization wavelength $\lambda_{0}=608.01 \mathrm{~nm}$. The two spectral signals demonstrate discrimination of both polarization modes resolved for a given length of the fibre sample. From the different values of the equalization wavelengths resolved for the same path length difference adjusted in the interferometer we can conclude that the sign of the group birefringence $G=N_{x}-N_{y}$ is negative.

The spectral signals recorded for the outer fibre cladding revealed that the equalization wavelength $\lambda_{0}$ can be resolved in the spectral range from 497 to $907 \mathrm{~nm}$ [13]. Similarly, the spectral signals recorded for the polarization modes revealed that the equalization wavelength $\lambda_{0}$ can be resolved in the spectral range from 536 to $766 \mathrm{~nm}$. In these spectral ranges, the wavelength dependence of the differential group index $\Delta N\left(\lambda_{0}\right)$ was measured. The measured values related to the outer cladding are shown in Fig. 6 by the crosses (denoted as SK222 glass) together with a polynomial fit. Similarly, the measured values related to the fundamental mode in the $X$ and $Y$ polarizations are shown by the crosses (denoted as $X$ mode and $Y$ mode) and demonstrates different dispersion slope for the polarization modes in the comparison with that for the SK222 glass. It should be noted here that an apparent discrimination between the spectral signals for the orthogonal polarizations was revealed in the measured spectral range, which indicates that the second holey fibre has higher group birefringence than the first one.

To measure the fibre group index $N\left(\lambda_{0 r}\right)$ at the reference equalization wavelength $\lambda_{0 r}$, the procedure presented in section 2.1. was used. For the outer fibre cladding, when $\lambda_{0 r}=649.54 \mathrm{~nm}$ was chosen, we adjusted $\Delta L\left(\lambda_{0 r}\right)=$ $14956 \mu \mathrm{m}$. Using $N_{r}\left(\lambda_{0 r}\right)=N_{o}\left(\lambda_{0 r}\right)=1.56247$ and Eq. (5), we obtain $N_{g}\left(\lambda_{0 r}\right)=$ 1.54317. Similarly, for the mode in the $X$ polarization, when $\lambda_{0 r}=649.54 \mathrm{~nm}$ was chosen, we adjusted $\Delta L\left(\lambda_{0 r}\right)=15180 \mu \mathrm{m}$. Using $N_{r}\left(\lambda_{0 r}\right)=1.56247$ and Eq. (5), we obtain $N_{x}\left(\lambda_{0 r}\right)=1.54730$. Moreover, using the procedure presented in section 2.3 , this value gives for the measured $\Delta L\left(\lambda_{0 r}\right)=214 \mu \mathrm{m}$ the 
cladding group index $N_{g}\left(\lambda_{0 r}\right)=1.54335$. From the values and the measured values of the differential group indices $\Delta N_{g}\left(\lambda_{0}\right), \Delta N_{x}\left(\lambda_{0}\right), \Delta N_{y}\left(\lambda_{0}\right)$, the group index $N_{g}\left(\lambda_{0}\right)$ of the outer fibre cladding and the group indices $N_{x}\left(\lambda_{0}\right), N_{y}\left(\lambda_{0}\right)$ of the polarization modes as a function of the equalization wavelength $\lambda_{0}$ were determined. The functions are represented in Fig. 7 by the crosses and are shown together with the polynomial fit for the cladding. This figure shows that the group indices of the polarization modes are substantially higher than the group index of the SK222 glass. According to Eq. (7), the group indices were measured with a precision of $13 \times 10^{-5}$.

\section{Conclusions}

We used a new white-light interferometric technique for measurement of the group index of holey fibres over a wide spectral range (in one case from 515 to $807 \mathrm{~nm}$ ). The technique utilized an unbalanced Mach-Zehnder interferometer with a fibre under test of known length placed in one of the interferometer arms and the other arm with adjustable path length. First, from a series of recorded spectral signals we measured the equalization wavelength as a function of the path length difference, or equivalently the differential group index dispersion of the fibre. Second, the fibre was replaced by the reference sample of known thickness and group dispersion and the group index of the fibre at one specific wavelength was determined precisely. The group index as a function of wavelength was measured for two different holey fibres, one made of pure silica glass and the other made of SK222 glass, and a precision was $13 \times 10^{-5}$.

The use of the method, whose main advantage is in the absolute group dispersion determination, can be extended for measuring cladding modes of holey fibres. Moreover, the sign of the group birefringence in highly birefringent holey fibres can be specified by the method. The method allows for the dispersion measurement in a wider spectral band, which can be further extended by applying the CCD array operating in another spectral range.

\section{Acknowledgements}

The research was partially supported by the Grant Agency of the Czech Republic (project no 102/06/0284), grant MSM6198910016 and internal grant of TU Ostrava (IGS HGF VŠB-TUO). 


\section{References}

[1] L.G. Cohen, J. Lightwave Technol. 3 (1985) 958.

[2] S Diddams, JC Diels, J. Opt. Soc. Amer. B 13 (1996) 1120.

[3] M Tateda, N Shibata, S Seikai, J. Quantum Electron. 17 (1981) 404.

[4] MJ Saunders, WG Gardner, J. Lightwave Technol. 5 (1987) 1701.

[5] P. Merritt, R.P. Tatam, D.A. Jackson, J. Lightwave Technol. 7 (1989) 703.

[6] F. Koch, S.V. Chernikov, J.R. Taylor, Opt. Commun. 175 (2001) 209.

[7] P. Lu, H. Ding, S.J. Mihailov, Meas. Sci. Technol. 16 (2005) 1631.

[8] J.Y. Lee, D.Y. Kim, Opt. Express 14 (2006) 11608.

[9] P. Hlubina, I Gurov, V Chugunov, J. Mod. Opt. 50 (2003) 2067.

[10] P. Hlubina, Opt. Commun. 218 (2003) 283.

[11] P Hlubina, T Martynkien, W Urbańczyk, Opt. Express 11 (2003) 2793.

[12] P. Hlubina, M. Szpulak, L. Knyblová, G. Statkiewicz, T. Martynkien, D. Ciprian, W. Urbańczyk, Meas. Sci. Technol. 17 (2006) 626.

[13] P. Hlubina, R. Chlebus, D. Ciprian, Meas. Sci. Technol. 18 (2007) 1547.

[14] D. Ouzounov, D. Homoelle, W. Zipfel, W.W. Webb, A.L. Gaeta, J.A. West, J.C. Fajardo, K.W. Koch, Opt. Commun. 192 (2001) 219.

[15] Q. Ye, C. Xu, X. Liu, W.H. Knox, M.F. Yan, R.S. Windeler, B. Eggleton, Appl. Opt. 41 (2002) 4467.

[16] L. Labonté, P. Roy, F. Pagnoux, F. Louradour, C. Restoin, G. Mélin, E. Burov, J. Opt. A: Pure Appl. Opt. 8 (2006) 933.

[17] P. Hlubina, M. Szpulak, D. Ciprian, T. Martynkien, W. Urbańczyk, Opt. Express 15 (2007) 11073.

[18] R. Buczynski, Acta Phys. Pol. 106 (2004) 141.

[19] P. Hlubina, Opt. Commun. 193 (2001) 1.

[20] P. Hlubina, D. Ciprian, L. Knyblová, Opt. Commun. 269 (2007) 8. 


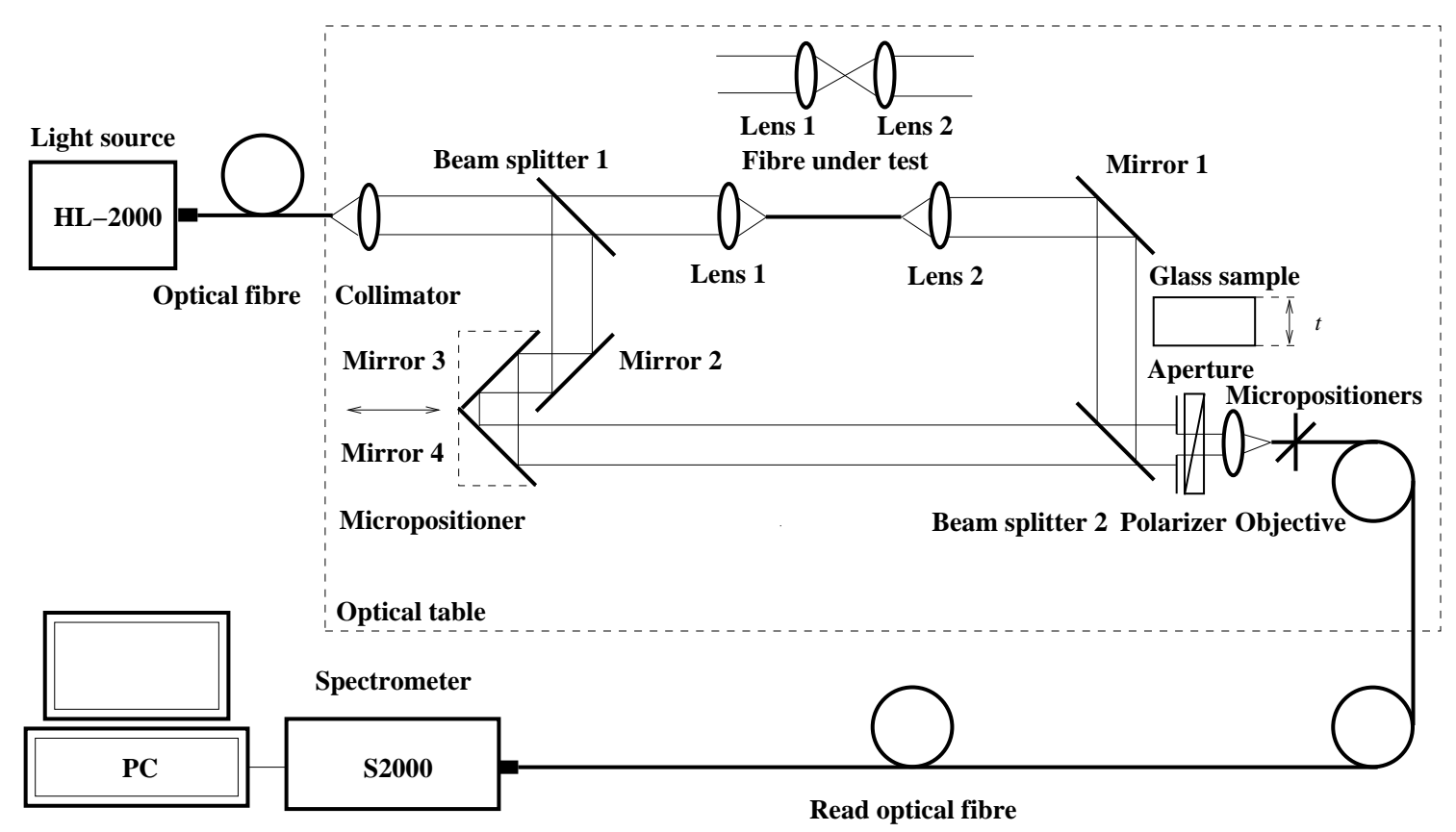

Fig. 1. Experimental set-up with an unbalanced Mach-Zehnder interferometer to measure the group index dispersion of a fibre under test. 


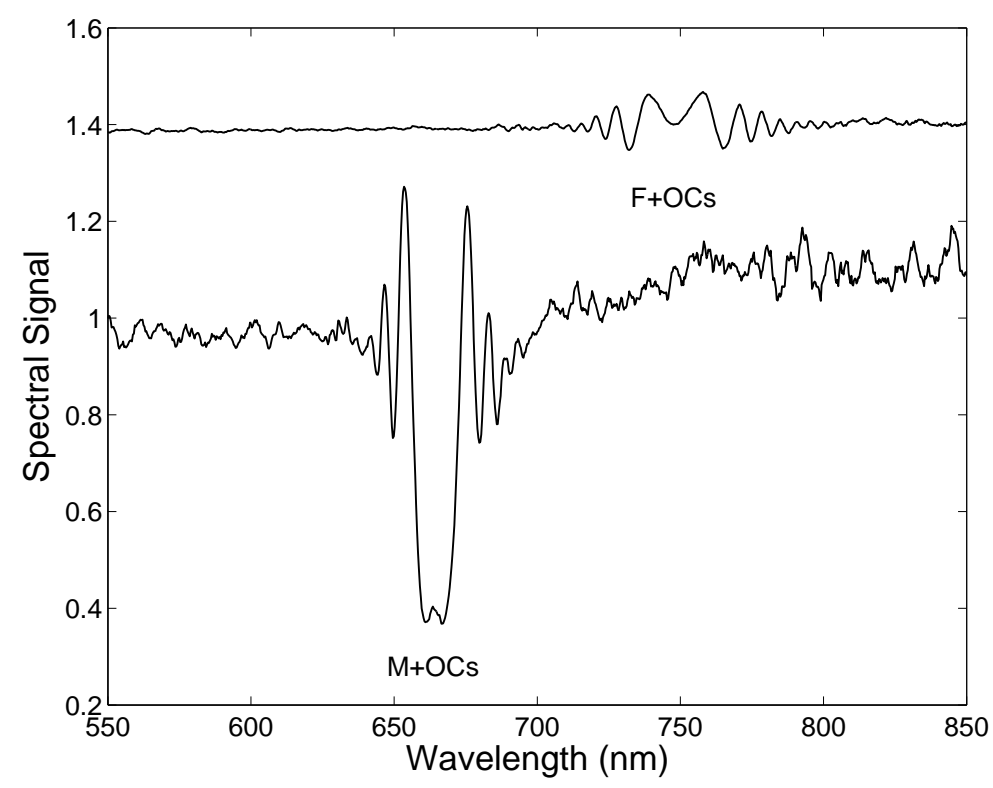

Fig. 2. Examples of the spectral signals recorded for two cases: outer fibre cladding plus optical components $(\mathrm{F}+\mathrm{OCs})$, fibre mode plus optical components $(\mathrm{M}+\mathrm{OCs})$.

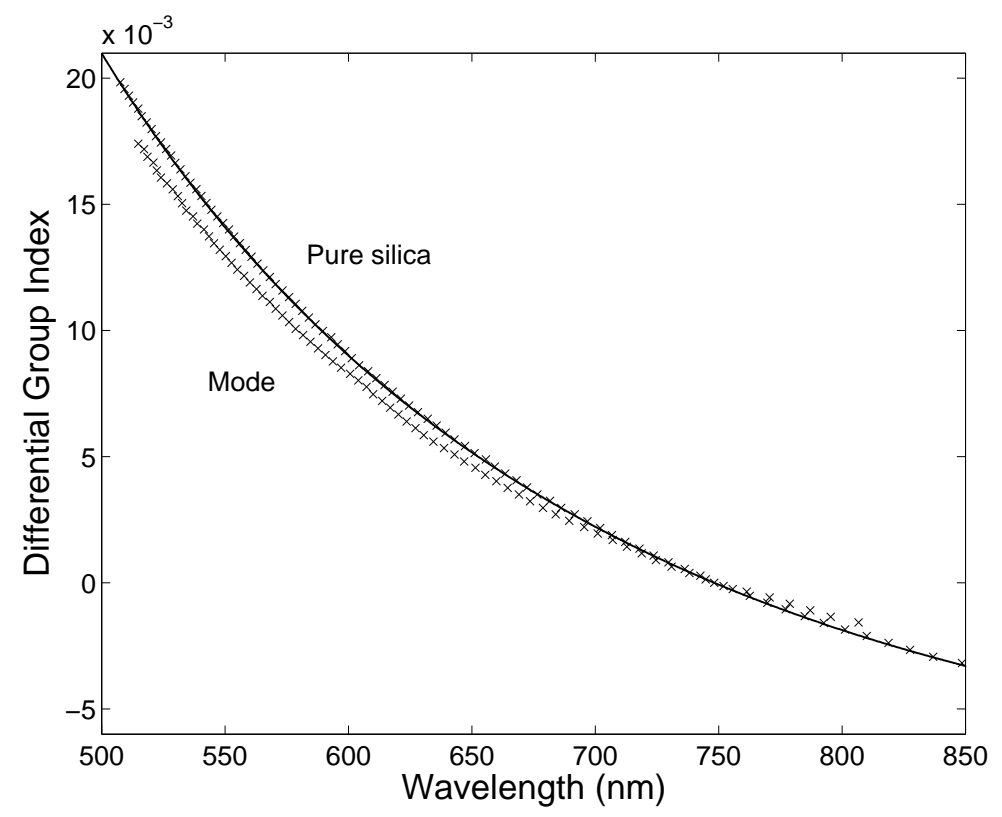

Fig. 3. Differential group index of outer fibre cladding (pure silica) and fibre mode measured as a function of wavelength. The solid line corresponds to theory. 


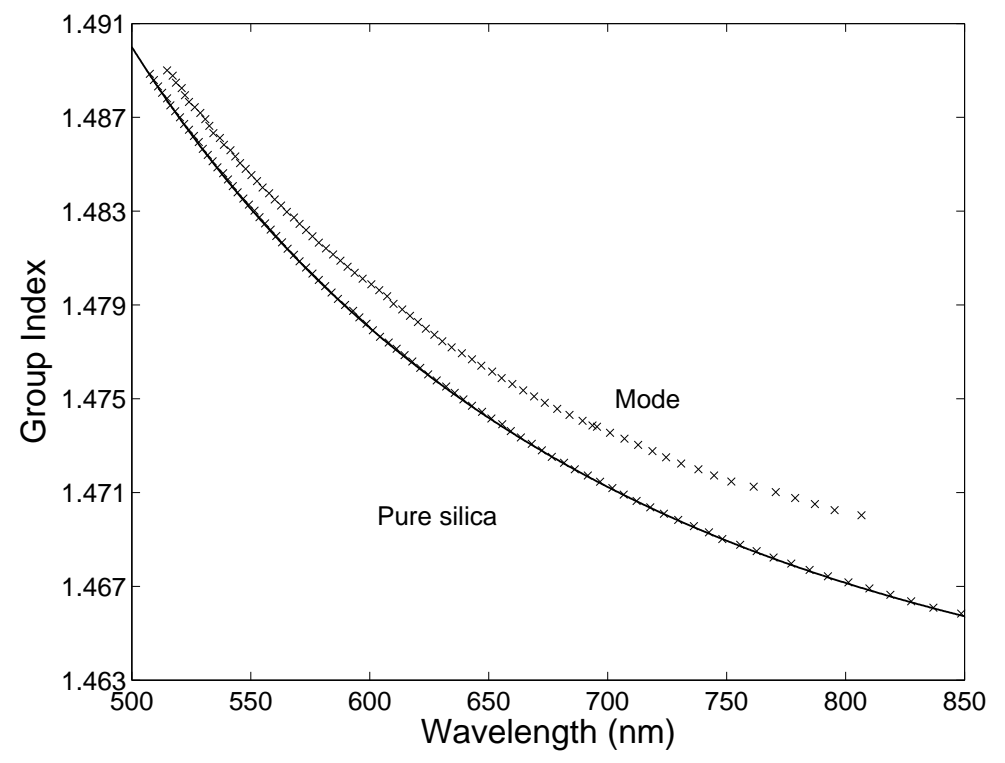

Fig. 4. Group index of outer fibre cladding (pure silica) and fibre mode measured as a function of wavelength. The solid line corresponds to theory.

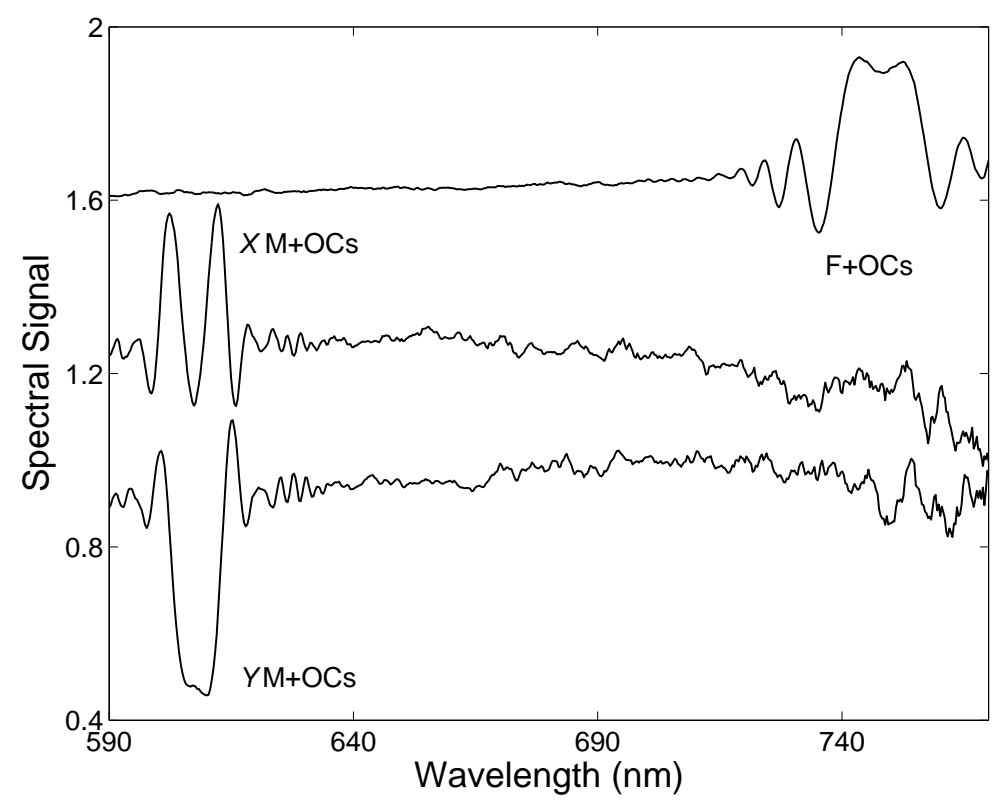

Fig. 5. Examples of the spectral signals recorded for two cases: outer fibre cladding plus optical components $(\mathrm{F}+\mathrm{OCs})$, fibre modes plus optical components $(X \mathrm{M}+\mathrm{OCs}$, $Y \mathrm{M}+\mathrm{OCs})$. 


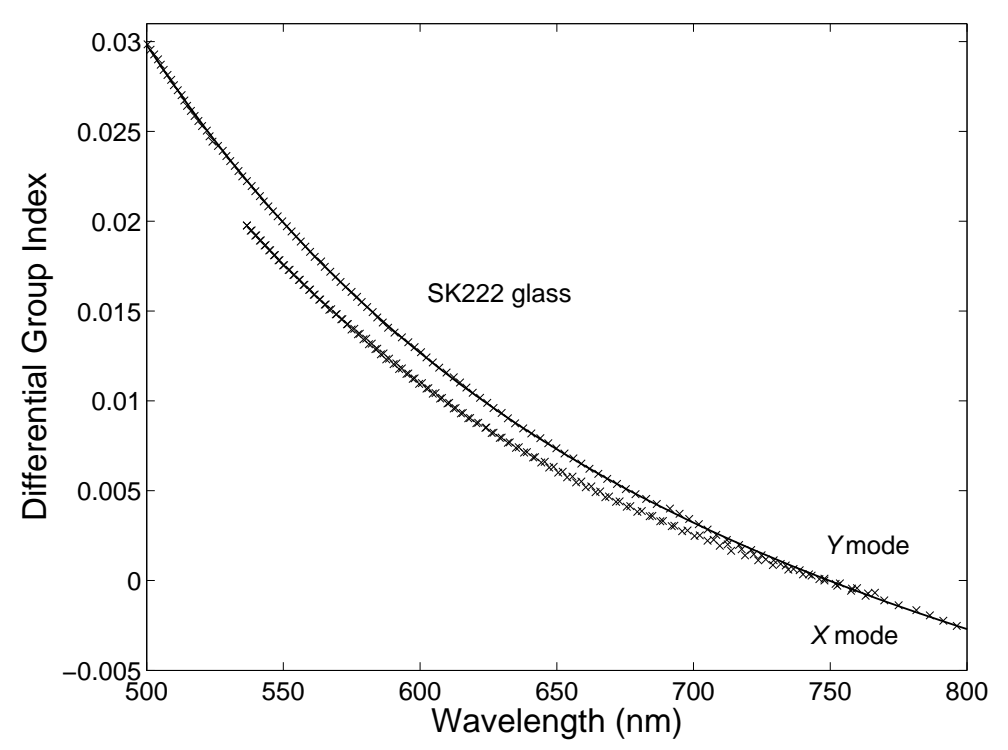

Fig. 6. Differential group index of outer fibre cladding (SK222 glass) and fibre modes measured as a function of wavelength. The solid line is a polynomial fit.

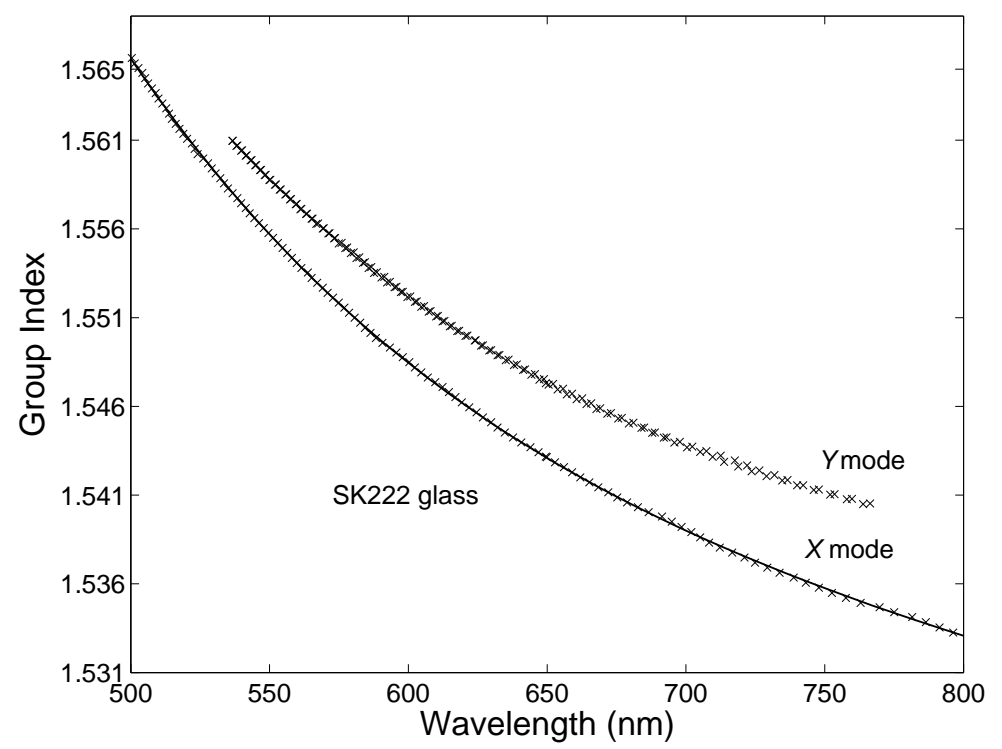

Fig. 7. Group index of outer fibre cladding (SK222 glass) and fibre modes measured as a function of wavelength. The solid line is a polynomial fit. 